\title{
A Clinical Prototype of the Digital Image Elasto Tomography Breast Cancer Screening System
}

\author{
Thomas Lotz* ${ }^{*}$ Amer Kashif*, Sheng Feng*, Pierrick Biret ${ }^{\dagger}$, Yohann Denais ${ }^{\dagger}$, Damien Lottin ${ }^{\dagger}$, \\ Laura Maillard ${ }^{\dagger}$, Thomas Tirschler ${ }^{\ddagger}$ and J Geoffrey Chase* \\ ${ }^{*}$ Centre for Bioengineering, Dept of Mechanical Engineering \\ University of Canterbury \\ Christchurch, New Zealand \\ Email: thomas.lotz@canterbury.ac.nz \\ †Université de Technologie de Belfort Montbéliard (UTBM) \\ Belfort, France \\ ${ }_{\ddagger}^{\ddagger}$ Technische Universität Dresden \\ Dresden, Germany
}

\begin{abstract}
Digital Image Elasto Tomography (DIET) is a noninvasive breast cancer screening modality that induces vibrations into a breast and images its surface motion with digital cameras. Disturbances seen in motion patterns are caused by areas of higher stiffness within the breast, potentially tumors. Limited in-vivo trials have been performed with a first prototype with promising results. To enable larger scale trials, a more compact and robust clinical prototype of the DIET system is required. This paper describes the considerations and development steps involved in the design process of the second DIET prototype, including ergonomics, functionality and cost. The outcome is a very portable and ergonomic device that is suitable for larger scale trials, even in remote locations. If trials prove successful, the DIET system offers great potential for low cost and accessible breast cancer screening, as an adjunct to existing modalities.
\end{abstract}

\section{INTRODUCTION}

Breast cancer is a significant health problem around the world, accounting for over 400,000 deaths every year [1]. Early detection and intervention can greatly increase five year survival rates to over 95\% [2]. Screening programs in the developed world commonly use x-ray Mammography to screen eligible women in a target age group. In spite of the benefits, Mammography is increasingly controversial due to low compliance, poor sensitivity [3], accumulated radiation exposure [4], discomfort [5] and over-diagnosis [6].

A low cost and less invasive modality is required to better meet screening demands, particularly in less developed countries or rural areas that lack screening programs [1]. This is for example the case in China, where no organized screening program is available for the mostly rural population. Breast cancer incidence has increased by 38\% from 2000-2005 and this number is likely underestimated due to the small proportion of the population (5.7\%) captured in the cancer registry [7]. Furthermore, due to different breast physiology, Mammography does not perform well in Chinese women [7] and a more suitable method is desired.

The Digital Image Elasto Tomography (DIET) system is a breast cancer screening modality to image elastographic information of the breast tissue. The technology's key advantages compared to other screening modalities are that it is non-invasive, does not require breast compressions, is very portable, and low in operational cost. Additionally, its performance improves in smaller and denser breasts, in which Mammography performs poorly. These benefits could render this approach very suitable for large screening programs, particularly in remote areas or countries with limited access to screening.

DIET relies on the high contrast difference in elastic properties of $300 \%$ to $1500 \%$ between cancerous tissue and healthy fatty or fibro-glandular tissue in the breast [8], [9], [10]. This contrast is an order of magnitude larger than the 5-10\% radiodensity contrast imaged in x-ray mammography [2].

In a DIET system, sinusoidal vibrations of $5-100 \mathrm{~Hz}$ are applied to the breast and the resulting surface oscillations are captured in three dimensions (3D) by an array of digital cameras tracking fiducial markers [11], [12], [13], as shown in Figure 1. Areas of higher stiffness within the breast result in a different surface vibration response compared to healthy tissue. This response can be used to determine the probability and angular location of a potentially cancerous inclusion [14]. With this approach, $10 \mathrm{~mm}$ masses can be localized in silicone phantoms [15]. Further computations with Finite Element [16] or Boundary Element inverse problems [17] can help to more accurately localize the tumor and its size.

Initial limited in vivo results show promising results (see Figure 1) and confirm the effects seen in vitro [15]. To further validate the DIET system in vivo, a larger clinical validation is required, which requires a robust and automated prototype system. This paper presents the specifications of 


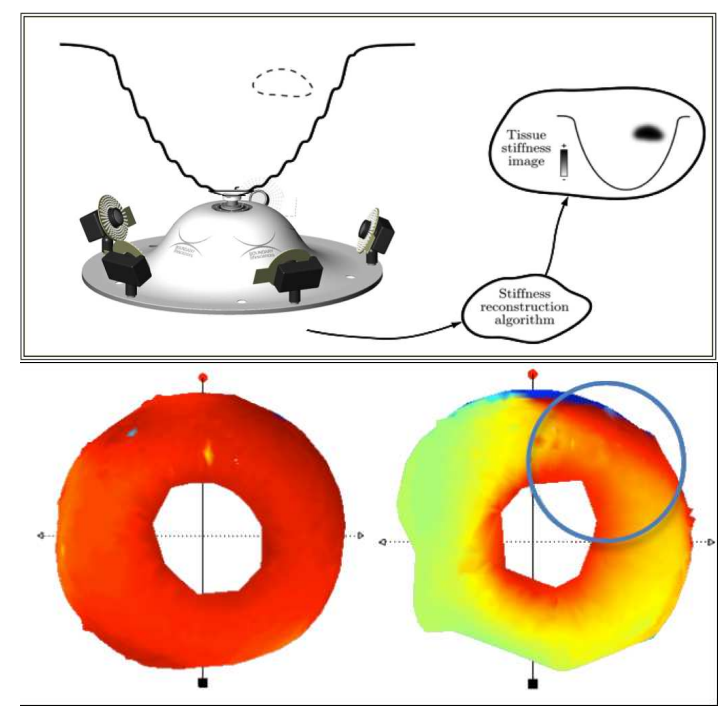

Fig. 1. Schematic of the imaging principle in DIET (top) and an in-vivo result showing the healthy (left) and cancerous breast (right) of the same woman. The circle indicates the location of the tumor.

such a prototype system and the development of the second DIET prototype intended for automated screening trials.

\section{DESIGN SPECIFICATIONS}

The design specifications are derived from experiences made on silicone phantoms and using the first clinical prototype in initial in vivo trials. This first prototype is functional but requires significant manual intervention and breast specific adjustments in the imaging procedure. This is not viable in a larger trial in which the system should be tested in parallel to a screening modality. Operational skill requirements should also be kept to a minimum. The operational aspects of the system are divided into the following categories:

1) Ergonomics - Overall design aspects of the interface to the body to allow comfortable imaging procedure and easy handling by staff

2) Hardware - Vibration actuator and additional positioning hardware

3) Imaging Array - Digital cameras suitable for fast and robust imaging

Figure 2 shows images of the first prototype. Identified improvements and requirements for the second clinical prototype are described in more detail in this section.

Computational processing of raw imaging results and diagnostic post-processing have not been included in this design project. These steps are performed on a processing unit separate to the DIET device and will have to be integrated at a later stage.

\section{A. Ergonomics}

The first DIET prototype has a flat surface on which a woman lies on with the torso, facing down (see Figure 2). This position is acceptable for a short time, but many trial subjects complained about neck pain and slight discomfort as
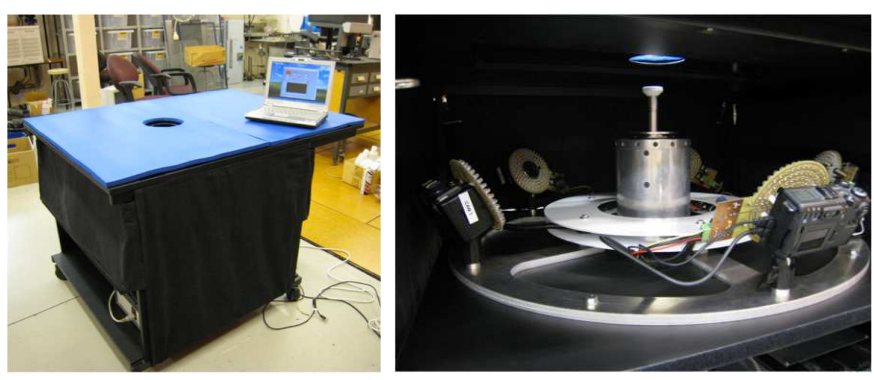

Fig. 2. First clinical prototype of the DIET system (left) and the actuator and imaging array within this prototype (right).

they had to lie still for the duration of the testing procedure, which was up to 30 minutes in some cases.

A further ergonomic aspect is system portability. The DIET system is intended to be portable and easy to operate, ideally by a single person if i.e. a remote mobile screening application is targeted. The current prototype is too bulky and heavy for this. The improvements and requirements regarding ergonomics are thus:

- Design an ergonomic surface that is comfortable for a woman to lie on. Physiological body shape considerations as well as functional aspects are to be considered in the design process.

- Design the overall prototype size and shape in such a way, that it is easily transportable by a single operator. The target is approximately the size of a suitcase, with a weight restriction of $25 \mathrm{~kg}$.

\section{B. Hardware}

The vibration actuator is a key component of the hardware system and is implemented using a voice coil that transfers a vertical oscillating vibration into the breast at the nipple area. Problems with the initial design include operational robustness and size. Particularly size is an issue to be addressed, as a smaller overall system will require an actuator with lower vertical dimensions.

During initial in vivo trials it also became clear that an automatic positioning system for the breast interface of the actuator is required. Varying breast physiology makes it impossible to define a generic centered position for the actuator underneath the breast. The two key design requirements for the vibration system in the second prototype are thus:

- Design a new vibration actuator with a smaller vertical height, i.e. no more than $120 \mathrm{~mm}$. The maximum dynamic actuator force required was identified to be $200 \mathrm{~N}$.

- Design a three axis positioning system to accurately place the breast interface at the nipple area of the breast. This subsystem should allow $40 \mathrm{~mm}$ stroke in horizontal $X$ and $\mathrm{Y}$ directions and $100 \mathrm{~mm}$ in vertical $\mathrm{Z}$ direction. Overall vertical height should be around $50 \mathrm{~mm}$ when at its lowest position.

\section{Imaging Array}

Imaging in the DIET system is done with an array of five digital cameras surrounding the breast. This ensures full 3D 
motion capturing of every point on the surface [12]. Currently, the imaging array is implemented with consumer digital cameras (Canon G9), which can be programmed and deliver good imaging quality. To reduce size and weight, and improve speed and automation, an array of much smaller industrial cameras is required in the second DIET prototype. A key requirement of the imaging array is low cost, as higher resolution industrial cameras can be very expensive. The aspects to be designed here include:

- Design an imaging array based on industrial CMOS or CCD cameras with a suitable resolution and image quality. Suitable lenses with sufficient depth of field in a fixed setup are to be sourced.

- Design a strobe flash system [18] with sufficient light intensity to achieve good image quality.

\section{Design Outcome}

\section{A. Ergonomics}

The ergonomics of the DIET system have been completely re-developed. The design is shown in Figure 3. Key elements of the new design include:

1) Shape of top surface adapted to female physiology

2) Integration of a head rest, similar to a massage table

3) Device size and weight are reduced and very portable
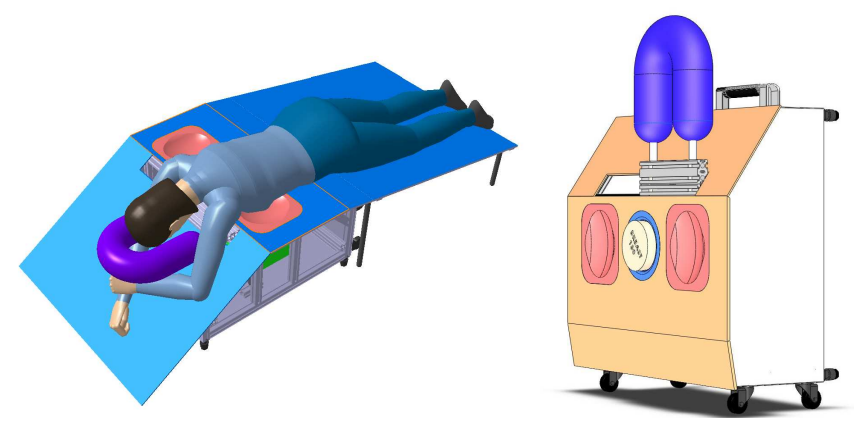

Fig. 3. Ergonomics of the second DIET clincial prototype with emphasis on comfort (left) and portability (right).

The objective of this design is to be modular and adaptive in changing environments, making it suitable for mobile screening applications. If required, the device could be placed on top of existing examination tables or on top of customized trolleys. Global dimensions and weight are kept small so that the device can be transported by a single person in a normal car. The core unit without the leg rests measures $0.7 \mathrm{~L} \times 0.6 \mathrm{~W} \times 0.35 \mathrm{H} \mathrm{m}$.

\section{B. Hardware}

The vibration system was re-developed to include a three axis positioning system and a more compact actuator, in a vertical build height smaller to the previous actuator alone, as shown in Figure 4. Key elements of the new design are:

1) The vibration actuator was designed using a more integrated approach to save space. Instead of using one large voice coil, three smaller coils are installed in parallel. The voice coils are now installed in between the flexure springs instead of underneath to save space. Overall actuator height could be reduced to $120 \mathrm{~mm}$.

2) A three-axis positioning device was developed that has $100 \mathrm{~mm}$ vertical stroke and compresses down to $60 \mathrm{~mm}$ in its lowest position. The $\mathrm{X}$ and $\mathrm{Y}$ dimensions are $320 \mathrm{~mm}$.
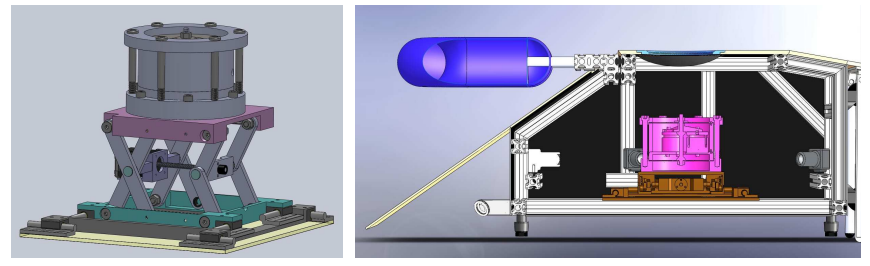

Fig. 4. Newly developed actuator and three axis positioning system (left) and inside view of overall prototype (right).

Actuator control is via a motor drive that powers all three voice coils in parallel, feedback controlled via a position sensor built into the actuator. The three-axis positioning system is controlled with visual feedback via the cameras to obtain an optimal position underneath the breast.

\section{Imaging Array}

The main objective of the imaging array was to implement industrial cameras that are much more compact and faster to operate than the consumer cameras currently used. Different cameras were evaluated, particularly to assess the tradeoff between price and imaging quality. Due to the fast motion imaging and high light sensitivity requirements, a global shutter CCD camera is likely more suitable than a rolling shutter CMOS sensor. Considering price differences of at least 2-3x between these sensors, alternative camera triggering options were evaluated to assess the feasibility of rolling shutter CMOS sensors. One identified and tested option is shown in Figure 5 for size comparison. The details are:

1) CMOS rolling shutter camera with $3 \mathrm{MP}$ resolution from IDS-Imaging (Obersulm, Germany).

2) A low aperture fixed focus lens to achieve high depth of field (i.e. F8).

3) Working distance of approximately $250-280 \mathrm{~mm}$ and a field of view of 200x150 mm.
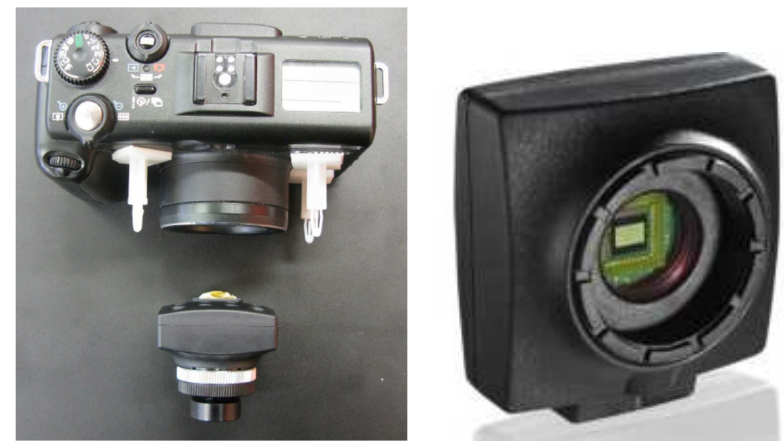

Fig. 5. Industrial camera evaluated for the DIET system (right) compared to the previously used Canon consumer camera (left). 


\section{Discussion AND Conclusions}

The Digital Image Elasto Tomography breast cancer screening technology has great potential for non-invasive and low cost screening programs. It is particularly aimed at developing countries with limited access to breast screening, due to remoteness, cost or both. In China, for example, where breast screening is paid privately, Infrared Thermography is a very popular imaging modality due to its low cost, despite its relatively poor reputation in many western world countries [19]. This shows that the development of ever more accurate imaging modalities at an increasing cost is only really an option for wealthy economies, and does not improve healthcare in most developing countries.

First DIET in vivo results are promising, but larger subject numbers are required for more detailed performance evaluations. The first clinical DIET prototype is functional but not practical for larger trials that require multiple imaging sites and possible frequent transportation and setup of a device. For this reason, a second, more portable and automated DIET prototype has been developed, incorporating technical improvements identified during initial trials.

The second DIET prototype includes an ergonomic patient interface and is easy to transport by a single person. Patient comfort has been reported as a key limiting factor in screening program compliance [5], so this aspect is quite important for acceptance of the technology. More improvements, particularly in weight will have to be addressed at a later stage, if a highly mobile screening trial is planned.

To ensure minimal operational skills of the system, a more automated imaging procedure is required. A key element of this feature is the three-axis positioning system implemented in the second prototype, that can position the breast interface of the actuator accurately. Visual feedback is via the built-in camera system.

Robust and fast imaging is achieved with an array of five industrial-style cameras. The tradeoff between imaging performance and cost needs to be assessed for every application. In this case, cost is a major driving factor, which led to the implementation of a lower cost CMOS camera. With camera prices expected to decrease in the future, the camera array could be easily updated to match demands.

Finally, a compact and automated DIET clinical prototype was designed, based on feedback from initial in-vivo trials using the first prototype. Technical and ergonomic improvements implemented are key factors in the success of system acceptance and its application in larger trials. Larger trials with more than one device are currently planned for mid-2011 in China and will provide more thorough validation of the DIET concept.

\section{ACKNOWLEDGMENT}

This work was supported by a Postdoctoral Fellowship grant (UOCX0807) from the NZ Foundation for Research, Science and Technology (FRST) and Boundary Lifesciences Ltd.

\section{REFERENCES}

[1] S. S. Coughlin and D. U. Ekwueme, "Breast cancer as a global health concern," Cancer epidemiology, vol. 33, no. 5, pp. 315-8, 2009.

[2] D. B. Kopans, Breast imaging, 2nd ed. Philadelphia: Lippincott Williams \& Wilkins, 1998.

[3] M. Kriege, C. T. Brekelmans, C. Boetes, P. E. Besnard, H. M. Zonderland, I. M. Obdeijn, R. A. Manoliu, T. Kok, H. Peterse, M. M. TilanusLinthorst, S. H. Muller, S. Meijer, J. C. Oosterwijk, L. V. Beex, R. A. Tollenaar, H. J. de Koning, E. J. Rutgers, and J. G. Klijn, "Efficacy of mri and mammography for breast-cancer screening in women with a familial or genetic predisposition," The New England journal of medicine, vol. 351, no. 5, pp. 427-37, 2004.

[4] R. Fazel, H. M. Krumholz, Y. Wang, J. S. Ross, J. Chen, H. H. Ting, N. D. Shah, K. Nasir, A. J. Einstein, and B. K. Nallamothu, "Exposure to low-dose ionizing radiation from medical imaging procedures," The New England journal of medicine, vol. 361, no. 9, pp. 849-57, 2009.

[5] A. Asghari and M. K. Nicholas, "Pain during mammography: the role of coping strategies," Pain, vol. 108, no. 1-2, pp. 170-9, 2004.

[6] P. C. Gotzsche, O. J. Hartling, M. Nielsen, J. Brodersen, and K. J. Jorgensen, "Breast screening: the facts-or maybe not," BMJ (Clinical research ed, vol. 338, p. b86, 2009.

[7] L. Yang, D. M. Parkin, J. Ferlay, L. Li, and Y. Chen, "Estimates of cancer incidence in china for 2000 and projections for 2005," Cancer epidemiology, biomarkers \& prevention, vol. 14, no. 1, pp. 243-50, 2005.

[8] A. Samani, J. Zubovits, and D. Plewes, "Elastic moduli of normal and pathological human breast tissues: an inversion-technique-based investigation of 169 samples," Physics in medicine and biology, vol. 52 , no. 6 , pp. $1565-76,2007$

[9] V. Egorov, S. Tsyuryupa, S. Kanilo, M. Kogit, and A. Sarvazyan, "Soft tissue elastometer," Medical engineering and physics, vol. 30, no. 2, pp. 206-12, 2008.

[10] T. A. Krouskop, T. M. Wheeler, F. Kallel, B. S. Garra, and T. Hall, "Elastic moduli of breast and prostate tissues under compression," Ultrason Imaging, vol. 20, no. 4, pp. 260-74, 1998.

[11] A. Peters, J. G. Chase, and E. E. Van Houten, "Estimating elasticity in heterogeneous phantoms using digital image elasto-tomography," Medical and biological engineering and computing, vol. 47, no. 1, pp. 67-76, 2009.

[12] R. Brown, C. Hann, J. Chase, and X. Chen, "Vision-based 3d surface motion capture for the diet breast cancer screening system," in 15th International Conference on Mechatronics and Machine Vision in Practice (M2VIP), Auckland, New Zealand, 2008 of Conference, pp. 674-679.

[13] R. Brown, C. Hann, J. Chase, and L. Ray, "Discrete colour-based euclidean-invariant signatures for feature tracking in a diet breast cancer screening system," in SPIE Medical Imaging, vol. 6511. San Diego, USA: SPIE, 2007 of Conference.

[14] S. Feng, T. Lotz, J. G. Chase, and C. E. Hann, "An image based vibration sensor for soft tissue modal analysis in a digital image elasto tomography (diet) system," in IEEE EMBS, vol. 1, Buenos Aires, 2010, pp. 25-8.

[15] T. Lotz, P. D. Simpson, D. Stocker, C. E. Hann, and J. G. Chase, "In vitro evaluation of surface based non-invasive breast cancer screening with digital image based elasto tomography (diet)," in IEEE EMBS, vol. 1, Buenos Aires, 2010, pp. 3077-80.

[16] A. Peters, J. G. Chase, and E. E. Van Houten, "Digital image elastotomography: combinatorial and hybrid optimization algorithms for shape-based elastic property reconstruction," IEEE transactions on biomedical engineering, vol. 55, no. 11, pp. 2575-83, 2008.

[17] H.-U. Berger, C. Hann, J. Chase, R. Broughton, and E. Van Houten, "Boundary element methods in elastography: A first explorative study," in SPIE Medical Imaging, vol. 6511. San Diego, USA: SPIE, 2007.

[18] C. Hann, J. Chase, X. Chen, C. Berg, R. Brown, and R. Elliot, "Strobe imaging system for digital image-based elasto-tomography breast cancer screening," IEEE Transactions on Industrial Electronics, vol. 56, no. 8, pp. 3195-3202, 2009.

[19] T. R. A. Radiologists and N. Z. C. of, "Royal australian and new zealand college of radiologists breast imaging reference group policy on the use of thermography to detect breast cancer," Tech. Rep., 2001. 